\title{
Antiviral and Antitumor Activity of Licorice Root Extracts
}

\author{
KUNIHIKO FUKUCHI ${ }^{1}$, NORIYUKI OKUDAIRA ${ }^{2}$, KAZUNORI ADACHI $^{2}$, \\ REINA ODAI-IDE ${ }^{2}$, SHIGERU WATANABE ${ }^{2}$, HIROKAZU OHNO ${ }^{3}$, MASAJI YAMAMOTO ${ }^{3}$, \\ TAISEI KANAMOTO ${ }^{4}$, SHIGEMI TERAKUBO ${ }^{4}$, HIDEKI NAKASHIMA ${ }^{4}$, \\ YOSHIHIRO UESAWA ${ }^{5}$, HAJIME KAGAYA ${ }^{5}$ and HIROSHI SAKAGAMI ${ }^{2}$ \\ ${ }^{1}$ Graduate School, Showa University, Tokyo, Japan; \\ ${ }^{2}$ Meikai University School of Dentistry, Sakado, Japan; \\ ${ }^{3}$ Maruzen Pharmaceuticals, Shibuya, Japan; \\ ${ }^{4}$ St. Marianna University School of Medicine, Kanagawa, Japan; \\ ${ }^{5}$ Meiji Pharmaceutical University, Kiyose, Japan
}

\begin{abstract}
Background: In the search for anti-viral and antitumor substances from natural resources, antiviral and antitumor activities of licorice root extract and purified ingredients were investigated. Materials and Methods: Viability of cells was determined by 3-14,5-dimethylthiazol-2yl)-2,5-diphenyltetrazolium bromide method. Antiviral activity was quantified by the selectivity index, defined as the ratio of the $50 \%$ cytotoxic concentration $\left(C C_{50}\right)$ to the $50 \%$ effective concentration against human immunodeficiency virus (HIV) or herpes simplex virus (HSV)-infected cells $\left(E C_{50}\right)$. The tumor specificity was calculated by the ratio of $\mathrm{CC}_{50}$ against human normal oral cells to that against human oral squamous cell carcinoma cell lines. Licorice flavonoids and lower molecular polyphenols were subjected to quantitative structure-activity relationship analysis. Results: Alkaline extract of licorice root had higher anti-HIV activity than did water extracts, confirming our previous reports. On the other hand, water extract, especially the flavonoid-rich fraction, had higher anti-HSV activity than did the alkaline extract. The flavonoid-rich fraction was more cytotoxic against human oral squamous cell carcinoma cell lines compared to normal oral cells, suggesting their tumor-specific cytotoxicity. Conclusion: The present study suggests that water and
\end{abstract}

This article is freely accessible online.

Correspondence to: Hiroshi Sakagami, Division of Pharmacology, Department of Diagnostic and Therapeutic Sciences, Meikai University School of Dentistry, Sakado, Saitama 350-0283, Japan. Tel: +81 492792758, Fax: +81 492855171, e-mail: sakagami@dent.meikai.ac.jp, kfukuchi@med.showa-u.ac.jp

Key Words: Licorice root, alkaline extract, fractionation, flavonoids, anti-HIV, anti-HSV, tumor-specificity, QSAR. alkaline extracts of licorice root exert different mechanisms of actions against these two viruses. Physicochemical properties, rather than the category of compounds, may be important in determining their anti-HSV activity.

We previously reported that lignin-carbohydrate complex fractions prepared by acid precipitation of the alkaline extracts of various plants potently protected cells from the cytopathic effect of human immunodeficiency virus (HIV) infection (1), and from the damage induced by UV irradiation (2). Crude alkaline extract of the leaves of Sasa senanensis Rehder (SE) $(3,4)$, green tea leaf, oolong tea leaf, orange flower $(5)$ and licorice root (6) had comparable anti-HIV and anti-UV activity to that of lignin-carbohydrate complex fractions $(3,4)$. On the other hand, hot-water extracts of various teas and orange flower (5) and Kampo medicines (7) exhibited much lower anti-HIV and anti-UV activity, suggesting the superiority of alkaline extraction over hot-water extraction in obtaining higher amounts of anti-HIV and anti-UV substances. We recently performed quantitative structure-activity relationship analysis of the cytotoxic activity of flavonoids derived from the licorice root against human oral malignant and nonmalignant cells (8). Although there are many articles on exist antiviral and antitumor activity of licorice products, most of them have not considered the chemotherapeutic index, which reflects both the cytotoxicity and efficacy. Furthermore, the antitumor activity of alkaline extract of licorice root has not yet been reported to our knowledge.

Based on the above, here we compared three aspects of biological activity (namely anti-HIV, anti-HSV and antitumor activity) of different licorice root extracts and purified components, according to the corresponding selectivity indices (SI). Polymethoxyflavonoids, including tricin, which has a broad antiviral spectrum (9-12), and SE, with potent anti-HIV and anti-HSV activity (13), were used as reference compounds. 


\section{Materials and Methods}

Materials. The following chemicals and reagents were obtained from the indicated companies: Dulbecco's modified Eagle's medium (DMEM) (Gibco BRL, Grand Island, NY, USA); fetal bovine serum (FBS), 3-(4,5-dimethylthiazol-2-yl)-2,5-diphenyltetrazolium bromide (MTT), RPMI-1640 medium, azidothymidine (AZT), 2',3'dideoxycytidine (ddC), resveratrol, melphalan (Sigma-Aldrich Inc., St. Louis, MO, USA); 3,3', 4', 5, 6,7,8-heptamethoxyflavone, nobiletin, tangeretin, sudachitin, methotrexate, dimethyl sulfoxide, dextran sulfate $(5 \mathrm{kDa})$ (Wako Pure Chemical Ind., Ltd., Osaka, Japan); curdlan sulfate (79 kDa; Ajinomoto Co. Inc., Tokyo, Japan); acyclovir, epigallocatechin gallate, curcumin, coumaric acid (Tokyo Chemical Industry Co. Ltd, Tokyo), tricin (Carbosynth ltd., Berkshire, UK). Glycyrrhizin acid and glycyrrhetinic acid were provided by Maruzen Pharmaceuticals (Shibuya, Tokyo, Japan). Culture plastic dishes and plates (96-well) were purchased from Becton Dickinson Labware (Franklin Lakes, NJ, USA). Bottled teas were obtained from a grocery store, and their osmotic pressures were measured by use of a cryoscopic osmometer (OSMOMAT030; Gonotec GmbH, Berlin, Germany).

SE was kindly provided by Daiwa Biological Research Institute Co., Ltd. (Kawasaki, Kanagawa, Japan). One milliliter of SE contained $66.1 \mathrm{mg}$ freeze-dried powder.

Preparation of water and alkaline extracts. Water and alkaline extracts of licorice root (Glycyrrhiza glabra harvested in Afghanistan) were prepared, as described previously (6).

Flavonoid and chalcone derivatives (liquiritin apioside, liquiritigenin 7-apiosylglucoside, liquiritin, neoliquiritin, liquiritigenin, isoliquiritin apioside, licurazid, isoliquiritin, neoisoliquiritin, isoliquiritigenin) were prepared from the $\mathrm{MeOH}$ extract of air-dried roots of $G$. inflate, by Diaion HP-20 and octadecylsilyl (ODS) column, as described previously (8).

Assay for anti-HIV activity. Human T-cell leukemia virus I (HTLVI)-bearing CD4- positive human T-cell line MT-4, established by Dr. Miyoshi (14) was cultured in RPMI-1640 medium supplemented with $10 \%$ FBS and infected with HIV-1 ${ }_{\text {IIIB }}$ at a multiplicity of infection of 0.01 . HIV- and mock-infected MT- 4 cells $\left(3 \times 10^{4}\right.$ cells/96-microwell $)$ were incubated for 5 days with different concentrations of extracts and the relative viable cell number was determined by MTT assay. The concentration that reduced the number of viable uninfected cells by $50 \%\left(\mathrm{CC}_{50}\right)$ and that which increased the number of viable HIVinfected cells to $50 \%$ that of control (mock-infected, untreated) cells $\left(\mathrm{EC}_{50}\right)$ were determined from the dose-response curve with mockinfected and HIV-infected cells, respectively. The anti-HIV activity was evaluated by the SI, which was calculated using the following equation: $\mathrm{SI}=\mathrm{CC}_{50} / \mathrm{EC}_{50}(15)$.

Assay for anti-HSV activity. We performed the MTT assay to quantify the anti-HSV activity of samples, as described previously (13). Vero cells $(10,000$ cells) were inoculated onto 96 -well plates (NUNC Labware Product-SigmaAldrich Inc, Tokyo, Japan). After $24 \mathrm{~h}$, the cells were infected with HSV-1 (supplied by National Institute of Infectious Disease Japan) (strain F) (multiplicity of infection=0.01). HSV-1 and test samples were mixed and stood for 20 min then added to the cells. After incubation for 4 days in 100 $\mu 1$ MEM-10\% fecal calf serum, MTT reagent (BioAssay Systems CA, USA) was then added and cells were incubated for a further 4 h. Cells were dissolved with $10 \%$ sodium dodecyl sulfate in $0.01 \mathrm{M}$ $\mathrm{HCl}$, and the absorbance at $595 \mathrm{~nm}$ was measured. The anti-HSV activity was evaluated by the SI (13). The $\mathrm{CC}_{50}$ was determined with mock-infected cells. EC $_{50}$ was determined by the following two methods, since the HSV infection did not achieve complete reduction of viability. Method 1 defined $\mathrm{EC}_{50}$ as the concentration at which the viability was restored to the midpoint between that of HSV-infected cells and that of mock-infected cells. Method II defined $\mathrm{EC}_{50}$ as the concentration at which the viability was restored to $50 \%$ that of mock-infected cells (Figure 1).

Assay for cytotoxic activity. Human normal oral cells [gingival fibroblast (HGF), periodontal ligament fibroblast (HPLF) and pulp cells (HPC)], established from the first premolar tooth extracted from the lower jaw of a 12-year-old girl (16), and human oral squamous cell carcinoma cell lines (Ca9-22, HSC-2, HSC-3, HSC4) purchased from Riken Cell Bank, Tsukuba, Japan were cultured at $37^{\circ} \mathrm{C}$ in DMEM supplemented with $10 \%$ heat-inactivated FBS, 100 units $/ \mathrm{ml}$, penicillin $\mathrm{G}$ and $100 \mu \mathrm{g} / \mathrm{ml}$ streptomycin sulfate under a humidified $5 \% \mathrm{CO}_{2}$ atmosphere. Cells were inoculated at $2.5 \times 10^{3}$ cells/0.1 $\mathrm{ml}$ in a 96-microwell plate (Becton Dickinson Labware). After $48 \mathrm{~h}$, the medium was removed by suction with an aspirator and replaced with $0.1 \mathrm{ml}$ of fresh medium containing different concentrations of single test compounds. Control cells were treated with the same amounts of vehicles, such as DMSO or ethanol, present in test solutions, and the cell damage induced by vehicles was corrected. Cells were incubated for a further $48 \mathrm{~h}$ and the relative viable cell number was then determined by the MTT method, as described previously (17). The $\mathrm{CC}_{50}$ was determined from the dose-response curve of triplicate assays.

The tumor-selectivity index (TS) was calculated by the following equation: $\mathrm{TS}=$ mean $\mathrm{CC}_{50}$ against normal cells/mean $\mathrm{CC}_{50}$ against tumor cells that is, D/B [HGF+HPLF+HPC (normal mesenchymal) (D) vs. Ca9-22+HSC-2+HSC-3+HSC-4 (tumor epithelial) (B)] or C/A [HGF (C) vs. Ca9-22 cells (A), derived from gingival tissue (18)] (Table III). We have confirmed that the TS value thus obtained reflects the antitumor activity although these normal and malignant cells were different types of cells from each other (either mesenchymal or epithelia), based on the finding that antitumor drugs have extremely higher TS values (19).

QSAR analysis. The $\operatorname{logSI}$ value was calculated from $-\log \mathrm{CC}_{50}$ value and - $\operatorname{logEC} 50$ value, as described previously (20). In order to determine the correlation coefficient, we used the data of method II and the following descriptors calculated by Dragon software ver 6.0.16 (Talete srl, Pisani, Milano, Italy): MATS5p (Moran autocorrelation of lag 5 weighted by polarizability; category, 2D autocorrelations) (21); GATS5p (Geary autocorrelation of lag 5 weighted by polarizability; category, 2D autocorrelations) (22); GATS5i (Geary autocorrelation of lag 5 weighted by ionization potential; category, 2D autocorrelations) (21); NRS (number of ring systems; category, Ring descriptors) (23); J_Dz(Z) (Balaban-like index from Barysz matrix weighted by atomic number; category, $2 \mathrm{D}$ matrix-based descriptors) (24); and J_Dz(m) (Balaban-like index from Barysz matrix weighted by mass; 2D matrix-based descriptors) (25).

Statistical treatment. Experimental values are expressed as the mean \pm standard deviation (SD). Statistical analysis was performed by using Mann-Whitney $U$-test. A value of $p<0.05$ was considered to be significant. The relation between anti-HSV activity and chemical 


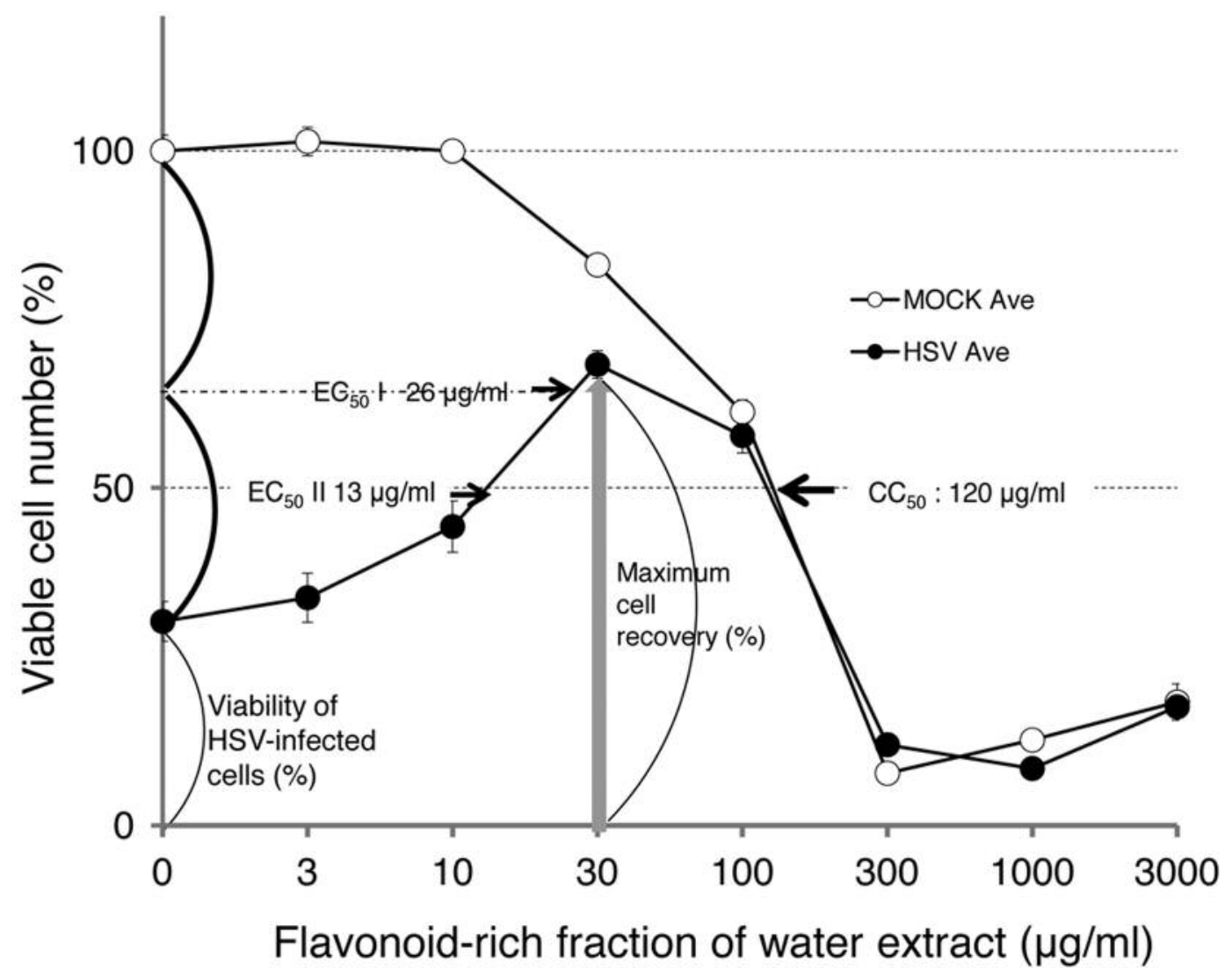

Figure 1. Anti-herpes simplex virus (HSV) activity of flavonoid-rich fraction of water extract of liquorice root. Each value represents the mean \pm SD of triplicate assays. The $50 \%$ effective concentration $\left(E C_{50}\right)$, determined by method I or II (see Table II), and $50 \%$ cytotoxic concentration $\left(C C_{50}\right)$ are indicated by arrows. HSV: Herpes simplex virus. Maximum cell recovery (\%) and viability of HSV-infected cells (\%) (Table II) are explained.

descriptors was investigated using simple regression analyses by JMP Pro version 12.2.0 (SAS Institute Inc., Cary, NC, USA).

\section{Results}

Anti-HIV activity. Infection of MT-4 cells with HIV-1 reduced viability to almost zero $(13,14)$. Among licorice extracts, water extract, its purified fraction and flavonoidrich fractions had no protective effect on HIV infection $(\mathrm{SI}<1)$ (Table I). Alkaline extract exhibited potent anti-HIV activity (SI>9.2), although the anti-HIV activity of alkaline extract was lower than that of SE $(\mathrm{SI}=42.7)$ and anti-viral agents (dextran sulfate, curdlan sulfate, AZT, ddC) $(\mathrm{SI}=3029-11715)$. The anti-HIV activity increased with increasing $\mathrm{pH}$ of the extracting solution $((\mathrm{SI}=><1,>3$ and $>9.2$ at $\mathrm{pH} 5.6,9$ and 12, respectively).

Glycyrrhizin acid, glycyrrhetinic acid, licorice flavonoids (iquiritin apioside, liquiritin 7-apiosylglucose, liquiritin, neoliquiritin, liquiritigenin, isoliquiritin apioside, lucurzid, isoliquiritin, neoisoliquiritin, isoliquiritigenin), tricin and other popular polymethoxyfavonoids $\left(3,3^{\prime}, 4^{\prime}, 5,6,7,8\right.$ - heptamethoxyflavone, nobiletin, tangeretin, sudachitin) were all inactive (SI $<1$ ), due to higher cytotoxicity (Table I).

Anti-HSV activity. Infection of Vero cells with HSV-1 reduced cell viability to $34.1 \pm 8.9 \%(18.3-51.1 \%) \quad(n=33)$ (Table II). This incomplete reduction of viability may be due to the slower spread of HSV infection than the growth of Vero cells.

We first confirmed that the addition of alkaline extract of the leaves of Sasa senanensis Rehder (SE) (used as positive control) to HSV-infected cells had potent anti-HSV activity $[\mathrm{SI}=6.0 \pm 2.7(3.6-9.3)(\operatorname{method} \mathrm{I})$ and $\mathrm{SI}=8.0 \pm 2.8(4.5-11.4)$ (method II)], increasing cell viability to $96.4 \pm 5.1 \%$ (93$100 \%$ ) (Table II). In contrast, licorice root extracts, licorice flavonoids, polymethoxyflavononoids, and low molecular weight polyphenols achieved significantly $(p<0.05)$ lower recovery of cell viability after HSV infection (viability of 51.0-64.0\%) (Table II). The lower recovery urged us to adopt two different methods for measuring the anti-HSV activity (method I and II). The higher anti-HSV activity of water 
Table I. Anti-human immunodeficiency virus (HIV) activity of licorice root extract. Each value was obtained by dose-response experiments of triplicate assays. The selectivity index (SI) was calculated using the following equation: $S I=C C_{50} / E C_{50}$, where $C C_{50}$ was the concentration that reduced the number of viable uninfected cells by $50 \%$ and $E_{50}$ was the concentration that increased the number of viable HIV-infected cells to $50 \%$ that of control (mock-infected, untreated) cells. SE: Alkaline extract of the leaves of Sasa senanensis Rehder; AZT: azidothymidine; ddC: 2',3'dideoxycytidine.

\begin{tabular}{|c|c|c|c|}
\hline & $\mathrm{CC}_{50}$ in mock-infected cells & $\mathrm{EC}_{50}$ in HIV-infected cells & SI \\
\hline \multicolumn{4}{|l|}{ Licorice root extracts } \\
\hline Water extract $(\mu \mathrm{g} / \mathrm{ml})$ & $>500$ & 387 & $>1.3$ \\
\hline Purfied fraction of water extract $(\mu \mathrm{g} / \mathrm{ml})$ & 61.09 & $>500$ & $<1$ \\
\hline Flavonoid-rich fraction of water extract $(\mu \mathrm{g} / \mathrm{ml})$ & 56.04 & $>500$ & $<1$ \\
\hline Water extract (different lot) $(\mathrm{pH} 5.6)(\mu \mathrm{g} / \mathrm{ml})$ & $>500$ & $>500$ & $><1$ \\
\hline Alkaline (pH 9.0) extract $(\mu \mathrm{g} / \mathrm{ml})$ & $>500$ & 165.79 & $>3.0$ \\
\hline Alkaine (pH12.0) extract $(\mu \mathrm{g} / \mathrm{ml})$ & $>500$ & 54.1 & $>9.2$ \\
\hline Glycyrrhizin acid $(\mu \mathrm{g} / \mathrm{ml})$ & $>500$ & 420.8 & $>1.2$ \\
\hline Glycyrrhetinic acid $(\mu \mathrm{g} / \mathrm{ml})$ & 47.84 & $>500$ & $<1$ \\
\hline \multicolumn{4}{|l|}{ Licorice flavonoids } \\
\hline Liquiritin apioside $(\mu \mathrm{g} / \mathrm{ml})$ & $>500$ & $>500$ & $><1$ \\
\hline Liquiritin 7-apiosylglucose $(\mu \mathrm{g} / \mathrm{ml})$ & $>500$ & $>500$ & $><1$ \\
\hline Liquiritin $(\mu \mathrm{g} / \mathrm{ml})$ & 28.82 & $>500$ & $<1$ \\
\hline Neoliquiritin $(\mu \mathrm{g} / \mathrm{ml})$ & 11.75 & $>500$ & $<1$ \\
\hline Liquiritigenin $(\mu \mathrm{g} / \mathrm{ml})$ & 10.2 & $>500$ & $<1$ \\
\hline Isoliquiritin apioside $(\mu \mathrm{g} / \mathrm{ml})$ & $>500$ & $>500$ & $><1$ \\
\hline Lucurzid $(\mu \mathrm{g} / \mathrm{ml})$ & $>500$ & $>500$ & $><1$ \\
\hline Isoliquiritin $(\mu \mathrm{g} / \mathrm{ml})$ & 9.6 & $>500$ & $<1$ \\
\hline Neoisoliquiritin $(\mu \mathrm{g} / \mathrm{ml})$ & 9.39 & $>500$ & $<1$ \\
\hline Isoliquiritigenin $(\mu \mathrm{g} / \mathrm{ml})$ & 3.86 & $>500$ & $<1$ \\
\hline \multicolumn{4}{|l|}{ Polymethoxyflavonoids } \\
\hline Tricin $(\mu \mathrm{M})$ & 354.71 & $>500$ & $<1$ \\
\hline $3,3^{\prime}, 4^{\prime}, 5,6,7,8$-Heptamethoxyflavone $(\mu \mathrm{M})$ & 64.58 & $>500$ & $<1$ \\
\hline Nobiletin $(\mu \mathrm{M})$ & 51.01 & $>500$ & $<1$ \\
\hline Tangeretin $(\mu \mathrm{M})$ & 130.77 & $>500$ & $<1$ \\
\hline Sudachitin $(\mu \mathrm{M})$ & 9.8 & $>500$ & $<1$ \\
\hline \multicolumn{4}{|l|}{ Positive control } \\
\hline $\mathrm{SE}(\%)$ & 1.11 & 0.026 & 42.7 \\
\hline Dextran sulfate $(\mu \mathrm{g} / \mathrm{ml})$ & $>1,000$ & 0.215 & $>4,651$ \\
\hline Curdlan sulfate $(\mu \mathrm{g} / \mathrm{ml})$ & $>1,000$ & 0.163 & $>6,135$ \\
\hline AZT $(\mu \mathrm{M})$ & 46.858 & 0.004 & 11,715 \\
\hline $\operatorname{ddC}(\mu \mathrm{M})$ & $2,198.73$ & 0.726 & 3,029 \\
\hline
\end{tabular}

extract of licorice root $[\mathrm{SI}=><1(\operatorname{method} \mathrm{I}), 4.6(\operatorname{method} \mathrm{II})]$ than alkaline extract $(\mathrm{pH} 12)[\mathrm{SI}<1(\operatorname{method} \mathrm{I}),<1$ (method II)] was unexpected. Flavonoid-rich fraction of water extract had the highest anti-HSV activity [SI=4.6 (method I), 9.2 (method II)]. Among licorice flavonoids, liquiritin apioside [SI $>5($ method I) $>500($ method II $)]$, isoliquiritin apioside [SI $>23.1$ (method I), >455 (method II)], lucurzid $[\mathrm{SI}><1$ $(\operatorname{method} \mathrm{I}),>667(\operatorname{method} \mathrm{II})]$ and isoliquiritin $[\mathrm{SI}=21.4$ (method I), 128.6 (method II)] had the highest anti-HSV activity.

Among five polymethoxyflavonoids, the SI value for tricin [SI=5.8 (method I), 7.0 (method II)] (Figure 1) was comparable with that of SE, while the other four polymethoxyflavonoids (3,3', 4', 5,6,7,8-heptamethoxyflavone, nobiletin, tangeretin, sudachitin) had little or no anti-HSV activity $[\mathrm{SI}<1$ (method I), <1 1.1 (method II)] (Table II).

Among lower molecular polyphenols, epigallocatechin gallate, a major compound in green tea, had some anti-HSV activity $[\mathrm{SI}<1$ (method I), 4.7 (mehod II)], followed by resveratrol $[\mathrm{SI}<1$ (method $\mathrm{I}), 2.8(\operatorname{method}$ II)] (Table II). This finding prompted us to survey the anti-HSV activity of commercially available bottles of tea extracts. Toasted tea (undiluted), oolong tea (10-fold diluted), green tea (commercial name: "tasty" tea) (undiluted), green tea (commercial name: "healthya" green tea) (10-fold diluted), black tea (commercial name: "afternoon tea") (10-fold 
Table II. Anti-herpes simplex virus (HSV) activity of licorice root extract. The selectivity index (SI) was calculated using the following equation: $S I=C C_{50} / E C_{50}$, where $C C_{50}$ was the concentration that reduced the number of viable uninfected cells by $50 \%$ and $E C_{50}$ was the concentration at which the viability was restored to the midpoint between that of HSV-infected cells and that of mock-infected cells (method I), or as the point where the viability was restored to 50\% that of mock-infected cells (method II). Each value was obtained by dose-response experiments of triplicate assays. ND: The SI value could not be determined due to the viability of HSV-infected cells exceeding 50\%. SE: Alkaline extract of the leaves of Sasa senanensis Rehder. Maximum cell recovery (\%) was calculated at the concentration that induced maximum recovery of viable cells. Maximum cell recovery (\%) and viability of $\mathrm{HSV}$-infected cells (\%) are explained in the legend of Figure 1.

\begin{tabular}{|c|c|c|c|c|c|}
\hline & & $\begin{array}{c}\mathrm{EC}_{50} \text { in HSV-infected } \\
\text { cells }\end{array}$ & & SI & \\
\hline $\begin{array}{l}\text { Viability of HSV- } \\
\text { infected } \\
\text { cells }(\%)\end{array}$ & $\begin{array}{c}\mathrm{CC}_{50} \text { in } \\
\text { mock- } \\
\text { infected cells }\end{array}$ & Method I Method II & Method I & Method II & $\begin{array}{c}\text { Maximum } \\
\text { cell recovery } \\
(\%)\end{array}$ \\
\hline
\end{tabular}

Licorice root extracts

Water extract $(\mu \mathrm{g} / \mathrm{ml})$

Purfied fraction of water extract $(\mu \mathrm{g} / \mathrm{ml})$

27.0

27.2

30.0

Flavonoid-rich fraction of water extract $(\mu \mathrm{g} / \mathrm{ml})$

Water extract (different lot) $(\mathrm{pH} 5.6)(\mu \mathrm{g} / \mathrm{ml})$

Alkaine (pH12.0) extract $(\mu \mathrm{g} / \mathrm{ml})$

Glycyrrhizin acid $(\mu \mathrm{g} / \mathrm{ml})$

Glycyrrhetinic acid $(\mu \mathrm{g} / \mathrm{ml})$

Licorice flavonoids

Liquiritin apioside $(\mu \mathrm{g} / \mathrm{ml})$

Liquiritin 7-apiosylglucose $(\mu \mathrm{g} / \mathrm{ml})$

Liquiritin $(\mu \mathrm{g} / \mathrm{ml})$

Neoliquiritin $(\mu \mathrm{g} / \mathrm{ml})$

Liquiritigenin $(\mu \mathrm{g} / \mathrm{ml})$

Isoliquiritin apioside $(\mu \mathrm{g} / \mathrm{ml})$

Lucurzid $(\mu \mathrm{g} / \mathrm{ml})$

Isoliquiritin $(\mu \mathrm{g} / \mathrm{ml})$

Neoisoliquiritin $(\mu \mathrm{g} / \mathrm{ml})$

Isoliquiritigenin $(\mu \mathrm{g} / \mathrm{ml})$

20.0

35.8

30.8

33.1

35.2

infected cells

$>3,000$
340
120
1,500
1,900
1,400
300
52

Alkaline ( $\mathrm{pH} 9.0)$ extract $(\mu \mathrm{g} / \mathrm{ml})$

(\%)

$\begin{array}{cc}>3,000 & 650 \\ 100 & 50 \\ 26 & 13 \\ >3,000 & 740 \\ >3,000 & 600 \\ >3,000 & >3,000 \\ >300 & >300 \\ >3,000 & >3,000\end{array}$

$><$
3.4
4.6
$<$
$<$
$<$
$<$
$<$

$>4.6$

60

$3.4 \quad 6.8$

$4.6 \quad 9.2$

$<1 \quad 2.0$

$<1 \quad 3.2$

$<1 \quad<1$

$<1 \quad<1$

$<1 \quad<1$

$\begin{array}{lcccccc}44.8 & >3,000 & 600 & 6 & >5 & >500 & 79 \\ 34.1 & >3,000 & >3,000 & 140 & ><1 & >21 & 58 \\ 33.8 & 14 & >3,000 & 5 & <1 & 2.8 & 55 \\ 41.6 & 52 & >3000 & 18 & <1 & 2.9 & 59 \\ 45.3 & 18 & 7.5 & <3 & 2.4 & >6 & 77 \\ 47.9 & >3,000 & 130 & 6.6 & 23.1 & >455 & 82 \\ 41.5 & >2,000 & >3,000 & 3 & ><1 & >667 & 65 \\ 35.9 & 18 & 0.84 & 0.14 & 21.4 & 128.6 & 97 \\ 20.0 & 37 & 4.6 & 3.4 & 8.0 & 10.9 & 87 \\ 25.2 & 12 & >100 & 6 & <1 & 2.0 & 54\end{array}$

Polymethoxyflavonoids

Tricin $(\mu \mathrm{M})$

$3,3^{\prime}, 4^{\prime}, 5,6,7,8$-Heptamethoxyflavone $(\mu \mathrm{M})$

18.3

28.2

Nobiletin $(\mu \mathrm{M})$

Tangeretin $(\mu \mathrm{M})$

Sudachitin $(\mu \mathrm{M})$

21.6

32.9

36.3

14

110

2.4

1,000

2
100

$>3,000>3,000$

$>3,000>3,000$

140

18

$>30$

5.8

$<1$

7.0
1.1

$<1$

$<1$

$<1$

50

69

57

62

49

ND

ND

Polyphenols

Epigallocatechin gallate $(\mu \mathrm{M}) \quad 29.3$

Chlorogenic acid $(\mu \mathrm{M})$

Coumaric acid $(\mu \mathrm{M})$

Curcumin $(\mu \mathrm{M})$

Resveratrol $(\mu \mathrm{M})$

29.3
33.3
41.5
51.1

70

2,000

$>300$

15

$>3,000$

$\begin{array}{ll}>3,000 & >3,000 \\ >3,000 & >3,000\end{array}$

10 ND

$>3000 \quad 7.4$

$\begin{array}{ll}<1 & 4.7 \\ <1 & <1\end{array}$

$<1$
$<1$

ND

2.8

50

$$
42
$$

45

42

Positive control

SE $(\%)(\mathrm{v} / \mathrm{v})$
SE $(\%)(\mathrm{v} / \mathrm{v})$
SE $(\%)(\mathrm{v} / \mathrm{v})$
SE $(\%)(\mathrm{v} / \mathrm{v})$
SE $(\%)(\mathrm{v} / \mathrm{v})$

diluted) and freshly made green tea (100-fold diluted) completely inhibited the cytopathic effect of HSV-infection, whereas barley tea was inactive (Figure 2).
Anti-tumor activity. Antitumor activity was determined by the ratio of cytotoxicity against four oral squamous cell lines vs. three normal oral cell types or the ratio of cytotoxicity against 
Table III. Tumor specificity (TS) of licorice root extracts. Each value was obtained by dose-response experiments of triplicate assays. HGF, Human gingival fibroblast; HPC, human pulp cells; HPLF, human periodontal ligament fibroblast; oral squamous cell carcinoma cell lines: Ca9-22 (derived from gingival tissue), HSC-2, HSC-3 and HSC-4 (derived from tongue); $\mathrm{CC}_{50}, 50 \%$ cytotoxic concentration; 5-FU, 5-fluorouracil.

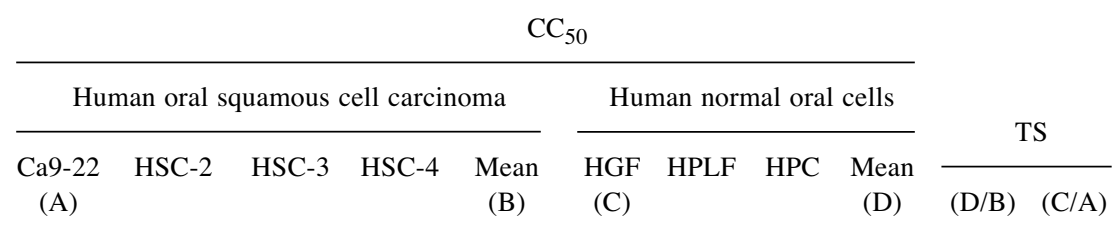

Licorice rool extracts

Water extract $(\mu \mathrm{g} / \mathrm{ml})$

Purfied fraction of water extract $(\mu \mathrm{g} / \mathrm{ml})$

Flavonoid-rich fraction of water extract $(\mu \mathrm{g} / \mathrm{ml})$

Water extract (different lot) $(\mathrm{pH} 5.6)(\mu \mathrm{g} / \mathrm{ml})$

Alkaline (pH 9.0) extract $(\mu \mathrm{g} / \mathrm{ml})$

Alkaine (pH12.0) extract $(\mu \mathrm{g} / \mathrm{ml})$

Glycyrrhizin acid $(\mu \mathrm{g} / \mathrm{ml})$

Glycyrrhetinic acid $(\mu \mathrm{g} / \mathrm{ml})$

Positive control

Doxorubicin $(\mu \mathrm{M})$

5-FU $(\mu \mathrm{M})$

Methotexate $(\mu \mathrm{M})$

Polymethoxyflavonoids

3,3', 4', 5, 6,7,8-Heptamethoxyflavone ( $\mu \mathrm{M})$

Nobiletin $(\mu \mathrm{M})$

Tangeretin $(\mu \mathrm{M})$

Sudachitin $(\mu \mathrm{M})$

Tricin $(\mu \mathrm{M})$

Doxorubicin $(\mu \mathrm{M})$

5-FU $(\mu \mathrm{M})$

Mephalan $(\mu \mathrm{M})$

Oil-soluble fraction of licorice

(G. glabra) $(\mu \mathrm{g} / \mathrm{ml})$

(G. uralensis) $(\mu \mathrm{g} / \mathrm{ml})$

(G. inflata) $(\mu \mathrm{g} / \mathrm{ml})$

Licorice flavonoids

Liquiritin apioside $(\mu \mathrm{g} / \mathrm{ml})$

Liquiritin $(\mu \mathrm{g} / \mathrm{ml})$

Neoliquiritin $(\mu \mathrm{g} / \mathrm{ml})$

Liquiritigenin $(\mu \mathrm{g} / \mathrm{ml})$

Isoliquiritin apioside $(\mu \mathrm{g} / \mathrm{ml})$

Isoliquiritin $(\mu \mathrm{g} / \mathrm{ml})$

Neoisoliquiritin $(\mu \mathrm{g} / \mathrm{ml})$

Neoliquiritin apioside $(\mu \mathrm{g} / \mathrm{ml})$

Neoisoliquiritin apioside $(\mu \mathrm{g} / \mathrm{ml})$

Isoliquiritigenin $(\mu \mathrm{g} / \mathrm{ml})$

Licochalcone A $(\mu \mathrm{g} / \mathrm{ml})$

Glabridin $(\mu \mathrm{g} / \mathrm{ml})$

Glycyrrhizin acid $(\mu \mathrm{g} / \mathrm{ml})$

Glycyrrhetinic acid $(\mu \mathrm{g} / \mathrm{ml})$

$\begin{array}{ccccccccccc}>500 & >500 & >500 & >500 & >500 & >500 & >500 & >500 & >500 & ><1 & ><1 \\ 103 & 200 & 69 & 153 & 131 & 438 & >500 & >431 & >456 & >3.5 & 4.2 \\ 51 & 75 & 35 & 71 & 58 & 263 & 347 & 351 & 320 & 5.5 & 5.2 \\ >500 & >500 & >500 & >500 & >500 & >500 & >500 & >500 & >500 & ><1 & ><1 \\ >500 & >500 & >500 & >500 & >500 & >500 & >500 & >500 & >500 & ><1 & ><1 \\ >500 & >500 & >500 & >500 & >500 & >500 & >500 & >500 & >500 & ><1 & ><1 \\ >500 & >500 & >500 & >500 & >500 & >500 & >500 & >500 & >500 & ><1 & ><1 \\ 77 & 61 & 50 & 68 & 64 & 86 & 65 & 63 & 71 & 1.1 & 1.1 \\ & & & & & & & & & & \\ & & & & & & & & & & \\ 0.28 & 0.09 & 0.13 & <0.078 & <0.15 & 3.47 & 9.57 & 0.45 & 4.50 & >30.9 & 12.2 \\ 35 & 208 & 10 & 198 & 113 & >1,000 & >1,000 & >1,000 & >1,000 & >8.9 & >28.8 \\ 8.97 & 0.19 & <0.13 & <0.13 & 2.35 & >400 & >400 & >400 & >400 & >170 & >45\end{array}$

$\begin{array}{ccccccccccc}110 & 176 & 81 & 238 & 151 & 305 & 317 & 295 & 306 & 2.0 & 2.8 \\ 91 & 189 & 50 & 182 & 128 & 165 & 306 & 302 & 258 & 2.0 & 1.8 \\ 131 & 100 & 117 & 91 & 110 & 310 & 365 & 313 & 329 & 3.0 & 2.4 \\ 40 & 158 & 56 & 117 & 93 & 67 & 500 & 252 & 273 & 2.9 & 1.7 \\ >500 & >500 & >500 & >500 & >500 & >500 & >497 & >500 & >499 & ><1.0 & ><1.0 \\ 0.133 & 0.060 & 0.090 & 0.058 & 0.085 & 7.3 & 1.3 & 9.3 & 6.0 & 69.9 & 54.8 \\ 92 & >1,000 & 128 & 79 & >325 & >1,000 & >1,000 & 234 & >745 & ><2.3 & >10.9 \\ 58 & 21 & 14 & 20 & 28 & 157 & 146 & 154 & 152 & 5.4 & 2.7\end{array}$

$\begin{array}{llllcllll}27 & 29 & 28 & 28 & 74 & 75 & 59 & 69.3 & 2.5 \\ 11 & 18 & 22 & 17 & 112 & 85 & 42 & 79.7 & 4.7 \\ 32 & 35 & 24 & 30 & 71 & 82 & 61 & 71.3 & 2.4\end{array}$

\begin{tabular}{|c|c|c|c|c|c|c|c|c|}
\hline$>200$ & $>200$ & $>200$ & $>200$ & $>200$ & $>200$ & $>200$ & $>200$ & $><1.0$ \\
\hline 156 & 69 & 128 & 118 & $>200$ & $>200$ & $>200$ & $>200$ & $>1.7$ \\
\hline$>200$ & $>200$ & $>200$ & $>200$ & $>200$ & $>200$ & 158 & $>186$ & $><0.9$ \\
\hline 38 & 49 & 86 & 58 & 145 & 138 & 70 & 118 & 2.0 \\
\hline$>200$ & 129 & $>200$ & $>176$ & $>200$ & $>200$ & $>200$ & $>200$ & $><1.1$ \\
\hline 43 & 163 & 73 & 93 & $>200$ & $>200$ & $>200$ & $>200$ & 2.2 \\
\hline 116 & $>200$ & 200 & $>172$ & $>200$ & $>200$ & 88 & $>163$ & $><0.9$ \\
\hline$>200$ & $>200$ & $>200$ & 200 & $>200$ & $>200$ & $>200$ & $>200$ & 1.0 \\
\hline 41 & 16 & 9.9 & 22 & $>200$ & $>200$ & $>200$ & $>200$ & 9.0 \\
\hline 4 & 9.5 & 9.9 & 7.8 & 42 & 37 & 25 & 34.7 & 4.4 \\
\hline 4.1 & 6.3 & 9.5 & 6.6 & 12 & 18 & 10 & 13.3 & 2.0 \\
\hline 8.5 & 8.9 & 8.8 & 8.7 & 22 & 21 & 11 & 18.0 & 2.1 \\
\hline$>200$ & $>200$ & $>200$ & $>200$ & $>200$ & $>200$ & $>200$ & $>200$ & $><1.0$ \\
\hline 27 & 29 & 36 & 31 & 59 & 68 & 30 & 52.3 & 1.7 \\
\hline 4.9 & 4.9 & 3.8 & 4.5 & 6.5 & 7.5 & 8.2 & 7.4 & 1.6 \\
\hline
\end{tabular}



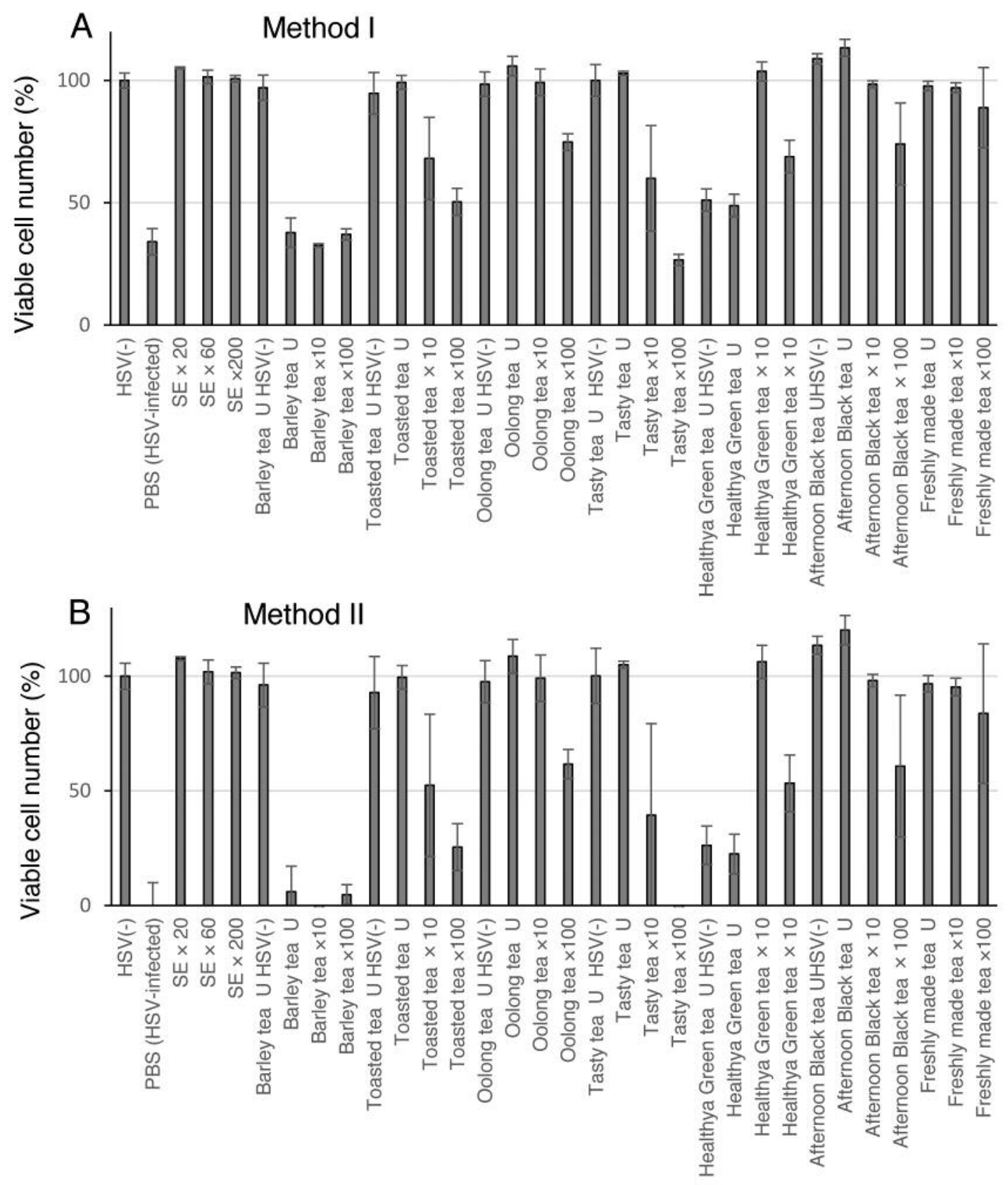

Figure 2. Anti-herpes simplex virus (HSV) activity of different tea extracts. The osmolarity of tea materials were adjusted to $280 \mathrm{mOsm}$ by adding solid $\mathrm{NaCl}$, filtered through Millipore filter to be sterilized prior to the use for experiments. The 50\% effective concentration (EC $\left.C_{50}\right)$ was the concentration at which the viability was restored to the midpoint between that of HSV-infected cells and that of mock-infected cells (method I) (A), or as the concentration at which the viability was restored to $50 \%$ that of mock-infected cells (method II) (B). Each value represents the mean $\pm S . D$. of triplicate assays. PBS, Phosphate-buffered saline; U, undiluted; $\times 10,10$-fold dilution, $\times 100,100$-fold dilution. SE $\times 20,60$ and 200 contained 3,1 , or $0.3 \mathrm{mg} / \mathrm{ml}$ dried materials, respectively.

Ca9-22 vs. HGF cells (both derived from gingival tissue) (Table III). Among licorice root extracts, flavonoid-rich fraction of water extract had the highest TS $[\mathrm{TS}=5.5 \mathrm{vs}$. normal oral cells, $5.2 \mathrm{vs}$. HGF cells], although this was much lower than that of popular antitumor drugs (doxorubicin, 5-fluorouracil, methotrexate, melphalan: TS=2.3-170). Oil-soluble fractions of licorice had moderate antitumor activity (TS=2.4-4.7). Among licorice flavonoids, neoisoliquiritin apioside and isoliquiritigenin 

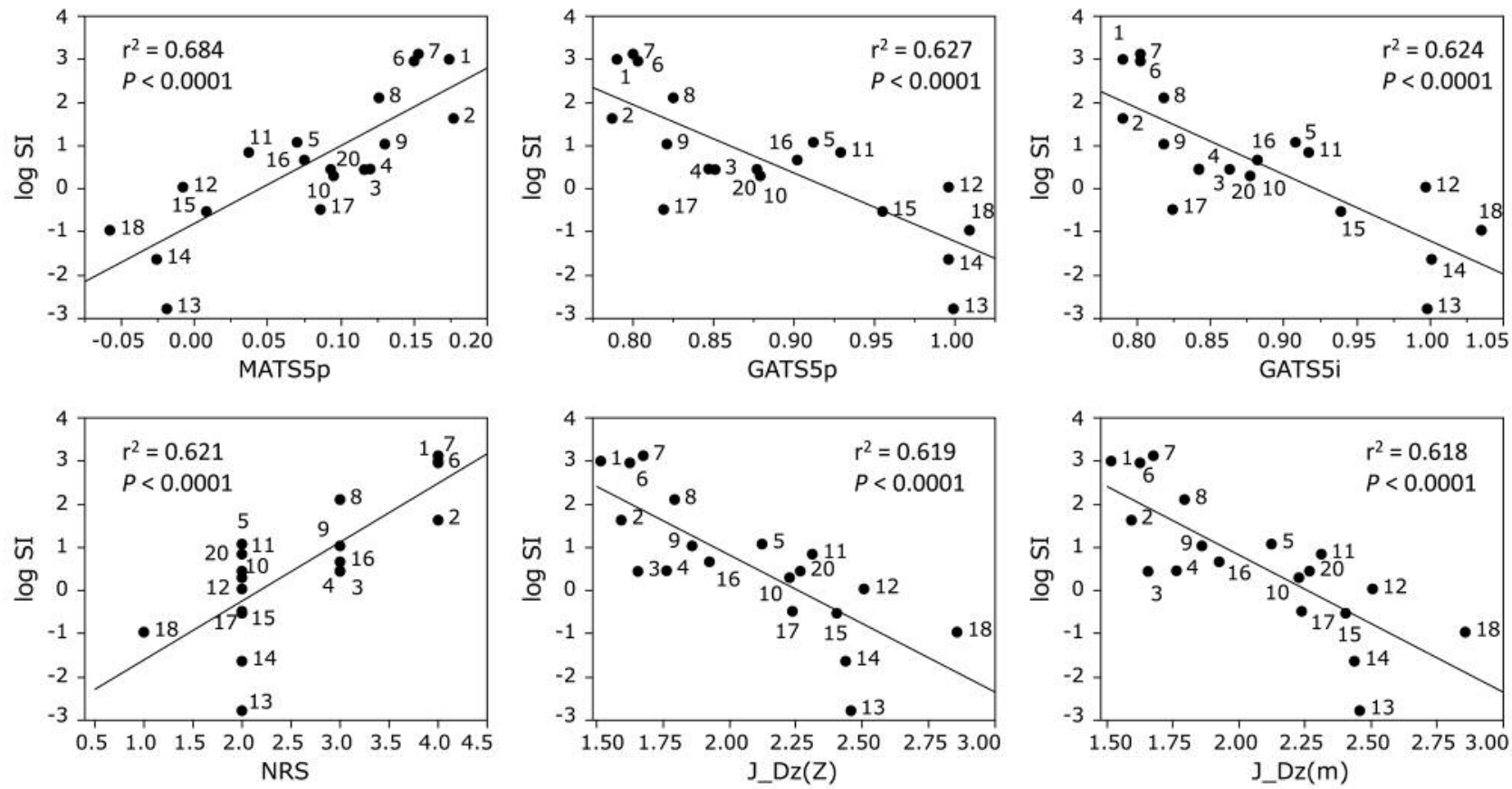

Figure 3. Determination of correlation between chemical descriptors and anti-herpes simplex virus (HSV) activity of licorice flavonoids, polymethoxyflavonoids and low molecular weight flavonoids (defined as selectivity index (SI) by method II in Table II). 1, Liquiritin apioside; 2, liquiritin 7-apiosylglucose; 3, liquiritin; 4, neoliquiritin; 5, liquiritigenin; 6, isoliquiritin apioside; 7, lucurzid; 8, isoliquiritin; 9, neoisoliquiritin; 10, isoliquiritigenin; 11, tricin; 12, 3,3',4',5,6,7,8-heptamethoxyflavone; 13, nobiletin; 14, tangeretin; 15, sudachitin; 16, epigallocatechin gallate; 17, chlorogenic acid; 18, coumaric acid; 20, resveratrol. The data of curcumin, 19, was not included since the SI value could not be obtained (Table II).

exhibited the highest antitumor activity (TS=4.4-9.0). Five polymethoxyflavonoids $\left(3,3^{\prime}, 4^{\prime}, 5,6,7,8\right.$-heptamethoxyflavone, nobiletin, tangeretin and sudachitin) were weakly tumor selective (TS=2.0-3.0) (Table III).

\section{Discussion}

The present study demonstrated that the alkaline extract of licorice roots had greater anti-HIV activity than the water extract (Table I), confirming our previous finding $(5,6)$, while the water extract, especially the flavonoid-rich fraction, had greater anti-HSV activity than the alkaline extract (Table II). This unexpected finding suggests the different mechanism of actions of water and alkaline extracts against these two viruses.

We next investigated the anti-HSV activity of licorice components. Licorice root flavonoids, especially liquiritin apioside, isoliquiritin apioside, lucurzid, isoliquiritin, showed higher anti-HSV activity than other polymethoxyflavonoids and low molecular weight polyphenols (Table II). We performed the preliminary QSAR analysis of anti-HIV activity using the mixtures of these 19 compounds. Among 1,705 chemical descriptors, six that represent polarizability (MATS5p, GATS5p) $(21,22)$, ionization potential (GATS5i) (22), number of ring systems (NRS) (24), atomic number
(J_Dz(Z)) and mass (J_Dz(m) (25) correlated well with antiHSV activity $\left(\mathrm{r}^{2}=0.684,0.627,0.624,0.621,0.619\right.$ and 0.618 , respectively, $p<0.0001$ ) (Figure 3 ). This result suggests that the physicochemical properties, rather than the category of compound, are important factors determining anti-HSV activity.

We also found that tricin, another four polymethoxyflavonoids and low molecular weight polyphenols incompletely overcame the cytopathic effects of HSV infection (Table II). It remains to be investigated whether combination of these compounds with acyclovir (15) or ganciclovir (25) may increase viability of infected cells.

The present study also demonstrated that neoisoliquiritin apioside had the highest anti-tumor activity. Isoliquiritin has been reported to inhibit granuloma angiogenesis and tube formation in vascular endothelial cells (26). Further studies are required to elucidate the mechanism by which this compound induces such high TS.

In conclusion, the present study suggests that alkaline extraction of licorice root may be applicable for the industrial mass-production of an anti-HIV agent, while the flavonoid-rich fraction of water extract might be used for the treatment of HSV. Further clinical study with licorice product is underway in our laboratory. 


\section{Conflicts of Interest}

This study was funded in part by Maruzen Pharmaceuticals (Hiroshi Sakagami), which caused no prejudice against the impartiality of the research reported.

\section{Acknowledgements}

This study was supported in part by from Grants-in-Aid for Scientific Research (C) (no. 16K11519, Principal investigator: Sakagami H) and the Miyata research fund B of Meikai University.

\section{References}

1 Sakagami H, Kushida T, Oizumi T, Nakashima H and Makino $\mathrm{T}$ : Distribution of lignin carbohydrate complex in plant kingdom and its functionality as alternative medicine. Pharmacol Therap 128: 91-105, 2010.

2 Nanbu T, Shimada J, Kobayashi M, Hirano K, Koh T, Machino M, Ohno H, Yamamoto M and Sakagami H: Anti-UV activity of lignin-carbohydrate complex and related compounds. In Vivo 27: 133-140, 2013.

3 Sakagami H, Sheng H, Okudaira N, Yasui T, Wakabayashi H, Jia J, Natori T, Suguro-Kitajima M, Oizumi H and Oizumi T: Prominent anti-UV activity and possible cosmetic potential of lignincarbohydrate complex. review. In Vivo 30(4): 331-339, 2016.

4 Sakagami H, Matsuta T, Yasui T, Oguchi K, Kitajima M, Sugiura T, Oizumi T and Oizumi T: Chapter 8, Functional evaluation of Sasa Makino et Shibata leaf extract as group III OTC drug. In: Alternative Medicine. Sakagami H (ed.), pp. 171-200. InTech, Croatia, 2012.

5 Sakagami H, Ohkoshi E, Amano S, Satoh K, Kanamoto T, Terakubo S, Nakashima H, Sunaga K, Otsuki T, Ikeda H and Fukuda T: Efficient utilization of plant resources by alkaline extraction. Altern Integr Med 2: 133, 2013.

6 Ohno H, Miyoshi S, Araho D, Kanamoato T, Terakubo S, Nakashima H, Tsuda T, Sunaga K, Amano S, Ohkoshi E, Sakagami H, Satoh K and Yamamoto M: Efficient utilization of licorice root by alkaline extraction. In Vivo 28: 785-794, 2014.

7 Kato T, Horie N, Matsuta T, Umemura N, Shimoyama T, Kaneko T, Kanamoto T, Terakubo S, Nakashima H, Kusama K and Sakagami H: Anti-UV/HIV activity of Kampo medicines and constituent plant extracts. In Vivo 26: 1007-1013, 2012.

8 Ohno H, Araho D, Uesawa Y, Kagaya H, Ishihara M, Sakagami $\mathrm{H}$ and Yamamoto M: Evaluation of cytotoxicity and tumorspecificity of licorice flavonoids based on chemical structures. Anticancer Res 33: 3061-3068, 2013.

9 Wang HK, Xia Y, Yang ZY, Natschke SL and Lee KH: Recent advances in the discovery and development of flavonoids and their analogues as antitumor and anti-HIV agents. Review Adv Exp Med Biol 439: 191-225, 1998.

10 Matsuta T, Sakagami H, Satoh K, Kanamoto T, Terakubo S, Nakashima H, Kitajima M, Oizumi H and Oizumi T. Biological activity of luteolin glycosides and tricin from Sasa senanensis Rehder. In Vivo 25(5): 757-62, 2011

11 Murayama T, Li Y, Takahashi T, Yamada R, Matsubara K, Tuchida Y, Li Z and Sadanari H. Anti-cytomegalovirus effects of tricin are dependent on CXCL11. Microbes Infect 14(12): 1086-1092, 2012.
12 Yazawa K, Kurokawa M, Obuchi M, Li Y, Yamada R, Sadanari H, Matsubara K, Watanabe K, Koketsu M, Tuchida Y and Murayama T. Anti-influenza virus activity of tricin, 4',5,7trihydroxy-3',5'-dimethoxyflavone. Antivir Chem Chemother 22(1): 1-11, 2011.

13 Sakagami H, Fukuchi K, Kanamoto T, Terakubo S, Nakashima H, Natori T, Suguro-Kitajima M, Oizumi H, Yasui T and Oizumi T: Synergism of alkaline extract of the leaves of Sasa senanensis Rehder and antiviral agents. In Vivo 30(4): 421-426, 2016.

14 Miyoshi I, Taguchi H, Kubonishi S, Yoshimoto S, Otsuki Y, Shiraishi $\mathrm{Y}$ and Akagi T: Type $\mathrm{C}$ virus-producing cell lines derived from adult $\mathrm{T}$ cell leukemia. Gann Monogr Cancer Res 28: 219-228, 1982.

15 Nakashima H, Murakami T, Yamamoto N, Sakagami H, Tanuma $\mathrm{S}$, Hatano $\mathrm{T}$, Yoshida $\mathrm{T}$ and Okuda $\mathrm{T}$ : Inhibition of human immunodeficiency viral replication by tannins and related compounds. Antiviral Res 18: 91-103, 1992.

16 Kantoh K, Ono M, Nakamura Y, Nakamura Y, Hashimoto K, Sakagami $\mathrm{H}$ and Wakabayashi H: Hormetic and anti-radiation effects of tropolone-related compounds. In Vivo 24: 843-852, 2010.

17 Sakagami H, Shimada C, Kanda Y, Amano O, Sugimoto M, Ota S, Soga T, Tomita M, Sato A, Tanuma S, Takao K and Sugita Y: Effects of 3-styrylchromones on metabolic profiles and cell death in oral squamous cell carcinoma cells. Toxocol Rep 2: 1281-1290, 2015.

18 Horikoshi M, Kimura Y, Nagura H, Ono T and Ito H: A new human cell line derived from human carcinoma of the gingiva. I. Its establishment and morphological studies. Jpn J Oral Maxillofac Surg 20: 100-106, 1974 (in Japanese).

19 Suzuki R, Matsuo S, Sakagami H, Okada Y and Shirataki Y: Search of new cytotoxic crude materials against human oral squamous cell carcinoma using NMR metabolomics. Anticancer Res 34(8): 4117-4120, 2014.

20 Uesawa Y, Sakagami H, Kagaya H, Yamashita M, Takao K and Sugita Y: Quantitative structure-cytotoxicity relationship of 3benzylidenechromanones. Anticancer Res 36, in press, 2016.

21 Moran PAP: Notes on continuous stochastic phenomena. Biometrika 37: 17-23, 1950.

22 Geary RC: The contiguity ratio and statistical mapping. Incorp Statist 5: 115-145, 1954.

23 Feher M and Schmidt JM: Property Distributions: Differences between drugs, natural products, and molecules from combinatorial chemistry. J Chem Inf Comput Sci 43: 218-227, 2003.

24 Ivanciuc O: QSAR comparative study of Wiener descriptors for weighted molecular graphs. J Chem Inf Comput Sci 40: 1412$1422,2000$.

25 Yamada R, Suda H, Sadanari H, Matsubara K, Tuchida Y and Murayama T: Synergistic effects by combination of ganciclovir and tricin on human cytomegalovirus replication in vitro. Antiviral Res 125: 79-83, 2016.

26 Kobayashi S, Miyamoto T, Kimura I and Kimura M: Inhibitory effect of isoliquiritin, a compound in licorice root, on angiogenesis in vivo and tube formation in vitro. Biol Pharm Bull 18(10): 1382-1386, 1995.
Received August 21, 2016

Revised September 13, 2016

Accepted September 15, 2016 